\title{
Freeway Travel Time Information from Input- Output Vehicle Counts: a Drift Correction Method Based on AVI Data
}

\author{
Francesc Soriguera* and Margarita Martínez-Díaz
}

\begin{abstract}
Input - output cumulative count curves are a powerful tool to forecast freeway travel times in the very short term, as they rely on the predictive information given by the vehicles' accumulation in the target section. Therefore, they could represent a very appealing method to feed real-time traffic information systems. However, traffic detectors' count drift implies very poor accuracy in the estimation of the vehicles' accumulation, leading to completely unreliable travel time predictions. A drift correction method is necessary. In contrast, several technologies allow accurate direct travel time measurements, like automatic vehicle identification (AVI) or vehicle tracking systems. In all cases, direct travel time measurements are obtained once the vehicle has crossed the target section. This means that the information is representative of the near past traffic conditions, while the objective of real-time information systems is to transmit information about traffic conditions in the near future. In this context, the present paper aims to fuse the information provided by input - output diagrams, obtained from loop detectors, with AVI direct travel time measurements. This fusion allows exploiting the accuracy of the direct measurements to correct the count drift in loop detectors. Then, corrected input - output curves can be used to obtain reliable short-term predictions of travel times. The proposed data fusion method has been applied to a test site in the AP7 freeway near Barcelona, obtaining significantly better results than with the common practices of simply disseminating direct measurements or using spot-speed methods.
\end{abstract}

Index Terms-Freeway travel time, cumulative count curves, loop detector drift, AVI technologies, data fusion, traffic information systems.

\section{INTRODUCTION AND BACKGROUND}

$\mathrm{S}_{\mathrm{a}}^{\mathrm{N}}$ NCE the earliest traffic volume measurements, more than century ago, the need for traffic data has grown together with the car fleet and the complexity of road networks. This tendency continues, and the envisioned cooperative and automated driving environments will rely on advanced traffic data. Fortunately, technological progress has also reached traffic surveillance. Today, surveillance systems are composed of a broad assortment of sensors and communication systems

Manuscript submitted to IEEE Transactions on ITS on March $25^{\text {th }}, 2019$. This work was supported in part by the Spanish Ministry of Economy and Competitiveness (Ministerio de Economía y Competitividad, Gobierno de España), id. TRA2016-79019-R/COOP. Publishing fees have been granted by the Agrupación Estratéxica CITEEC (Universidade da Coruña). capable of the real-time gathering and processing of huge amounts of traffic data. These have supported the rise of Advanced Traffic Management Systems (ATMS), Advanced Traffic Information Systems (ATIS), and Incident Management Systems (IMS) [1].

One of the key elements of ATIS is the travel time information, which is the best indicator of the level of service of a road link and the worthiest information for drivers. However, due to the existing surveillance heterogeneity, the availability of travel time information is limited and exhibits variable levels of accuracy, being, in many cases, not satisfactory. Current systems use various sources of information. On the one hand, direct travel time measurements can be obtained either from AVI (Automated Vehicle Identification) technologies or from tracking. The case of AVI systems is straightforward: each individual vehicle is identified at two control points, which delimit a section of the road. Its travel time in the section is calculated as the difference between the vehicle's time stamps at both locations. Examples of AVI technologies include Bluetooth recognition [2], toll tag identification [3], [4], [5], license plate automatic recognition (LPR) [6], [7], or the use of traditional inductive loop detectors to identify individual vehicles based on several distinctive characteristics such as their length [8], [9], [10] or their inductance signature [11], [12], [13]. Furthermore, individual vehicle identification is very useful, for example, for the estimation of $\mathrm{O} / \mathrm{D}$ matrices. The inconveniences of low penetration rates and high costs of these technologies in the past, have largely been overcome. However, some issues remain. First, travel time measurements obtained from AVI systems are captive of the control points, i.e., they are only available for the sections delimited by the sensors. Neither partial, nor longer trips can be measured. Second, travel time measurements in a given section are obtained once vehicles have completely covered it. This is the reason why these measurements are called arrival-based travel times (ATT), and represent a somehow outdated information for vehicles entering the section, especially if this section is long, or when congestion exists.

*Corresponding Author.

F. Soriguera is with the Barcelona Innovative Transportation (BIT) research group, UPC - BarcelonaTech, 08034 Barcelona (Spain).

M. Martínez-Díaz is with Universidade da Coruña, E.T.S.I. Caminos, Canales y Puertos, 15071 A Coruña (Spain). 
Tracking technologies represent an alternative to AVI for the direct measurement of travel times. These systems can provide individual vehicles' trajectories every few seconds, so that travel times between any two points on the highway can be obtained. Examples of tracking technologies include GPS, either in vehicles' navigation devices or in smartphones [14], phone signal geopositioning [15] or video surveillance using drones [16]. In fact, these technologies represent the evolution of traditional probe cars and are expected to be the prevailing source of traffic information in the future. Travel time measurements provided by tracking technologies are generally called instantaneous travel times (ITT), as they are based on the last information available. Still, ITT do not provide the desired information to drivers starting their trips, as they do not include a prediction about the conditions that these vehicles will face in the near future.

On the other hand, it is possible to indirectly estimate travel times from other traffic variables such as speeds or flows. Actually, this is the most common approach at traffic management centers, as the necessary inputs can be obtained from inductive-loop detectors, which continue being the most widespread surveillance technology. The usual methodology, the so-called "spot-speed method", obtains the travel time between two consecutive loops from the estimated average speed in the section. This average speed is computed by extrapolating the punctual speed measurements at detectors' locations. A high density of double loops (single loops do not provide accurate speed measurements), ideally a loop every 500 $\mathrm{m}$, is necessary to obtain acceptable results. Still, large errors can appear in congestion or in traffic state transitions [17]. One possibility to improve spot-speed methods was developed by [18] and [19]. They suggested a procedure to account for traffic dynamics in the speed spatial interpolation between measurements. This approach could be used in low surveillance environments, increasing the accuracy of the travel time estimation without a huge investment in intensive monitoring. However, it can only be applied when all the section between detectors is either free flowing or fully congested, as traffic state transitions are overlooked. Because the lengths of these sections can be of several kilometers, the fact of not considering the frequent traffic transitions implies significant errors. In any case, spot-speed methods yield ITT estimations, without predictive capabilities.

\section{\# FIGURE 1 \#}

A potentially useful method to compute travel times from traditional loop detector measurements is the use of cumulative input-output count curves together with the vehicle conservation equation [20], [21], [22]. The advantages of inputoutput methods are twofold: first, they can be applied in low surveillance environments and use data from the already installed detectors, which can be simple loops, as only vehicle counts are necessary. By low surveillance it is meant that intensive monitoring (i.e. closely spaced double-loop detectors; see Fig 1a) is not necessary, although some minimum monitoring requirements exist. For example, the target section needs to be "closed", with all the input/output flows measured to ensure vehicle conservation in data. This means that loop detectors must be installed in all on/off ramps (see part b1 of Fig. 1). Alternatively, if ramps are not monitored, detectors in the main trunk might suffice, if they are placed just before and after each junction (see Part b2 of Fig. 1). In this last case travel times are informed to/from the junction, while the short intrajunction distance is neglected.

The second main advantage of input-output methods is that they are based on the measurement of vehicles' accumulation between detectors. This provides information about the expected evolution of travel times in the short term, and can yield predicted travel times (PTT), a key feature in real-time information systems. None of the previous methods presents such predictive capabilities, and all of them provide, to some extent, a past measurement. For instance, in travel time evolving conditions, measured ATT would be completely flawed as real-time information, especially if the target stretch is long. Note that the delay in the information grows with the distance between AVI devices. Also, input-output counts can be updated more frequently than ATT measurements based on AVI data, because the aggregation periods of direct measurements are necessarily longer in order to obtain a sufficient sample size. And finally, partial travel times within the stretch can be obtained as, frequently, the density of loop detectors is higher than that of AVI devices.

In spite of all these advantages, input-output methods are not currently used for freeway travel time estimation. Not being an intuitive method or the need for closed detector configurations may contribute to this situation. Nevertheless, the most problematic factor is that loop detectors suffer from drift (i.e. small counting errors). Detector drift is not an important problem when data is analyzed for an isolated detector. However, in input-output methods, where the accumulation is computed from the relative difference between the cumulative counts at consecutive detectors, even a small drift accumulated over time can lead to meaningless results. To the authors' best knowledge, there is not any significant research work aimed at understanding and solving the undesirable loop detector drift for travel time estimation, yet. Therefore, there is a need for additional efforts to develop an adequate real-time methodology that $i$ ) is aimed at the prediction of travel times from cumulative count curves obtained from traditional loop detectors on low surveillance highway stretches, ii) performs detector drift correction, iii) accounts for typical difficulties in input - output methods like the existence of inner junctions in the closed sections or the effects of passing.

The present paper addresses these objectives by proposing a data fusion method. Data fusion arises as a promising choice, as it has already been proved useful in traffic state estimation [23], [24], [25], [26] and even in travel time prediction [27], [28], [29]. The method proposed here fuses data from traditional loops with AVI direct travel time measurements. Although not specifically addressed in the present paper, vehicle tracking data could also be used as the source for direct measurements, instead of AVI. In any case, because in general the spatio-temporal aggregation and coverage of the different 
data sources is not uniform, the method needs to include the spatial and temporal data alignment as a first fusion step. Then, direct measurements are used to correct the drift in the cumulative curves constructed from detector counts. From these corrected input-output curves, predicted travel time (PTT) estimations are obtained. This allows exploiting the predictive capabilities of input-output methods while keeping the accuracy in the estimation. Note that in the proposed data fusion scheme, direct measurements will be assumed as past ground truth values. This approach considers that the sampling errors of direct measurements can be neglected in front of the much larger uncertainty affecting the resulting predicted travel times. Also, because direct measurements are not used as the final information, a low penetration of the measurement technology, resulting in periods with few or any data, does not imply extremely severe drawbacks. In spite of this, if direct measurements include a large systematic bias, they will inevitably affect the results. In such case, however, disseminating the directly measured travel times would not produce better results.

Before moving forward, it should be understood that data fusion is not the only methodological option to accomplish the proposed objective (i.e. to obtain short-term predicted travel times). In particular, Computational Intelligence (CI), in any of its multiple classes, could be an alternative. CI is becoming increasingly popular in scientific studies, mainly due to the democratization of computational power and to the development of software packages allowing an easy implementation of the complex CI methods. In the field of travel time prediction, take as references [30] using state-space neural networks (SSNN) or [31] using several classes of deep learning techniques. In this context, the aim of the present paper is not to argue only and fervently in favor of the proposed approach, but to provide an alternative. Statistical methods based on the inherent causalities of the problem (as the one proposed here) imply pros and cons with respect to the more data analytic CI methods ([32] provides an excellent discussion on this topic). Selecting the proper methodology should not only be a matter of accuracy, as many other factors affect the results (i.e. time and effort for model development, skills and expertise required, transferability, adaptability, etc.). In this sense, the existence of several alternatives may result useful and generally, they can be combined to exploit the existing synergies.

The structure of the remainder of the paper is as follows: next, Section 2 reviews the main concepts in the input-output method to estimate travel times. Section 3 presents the proposed data fusion algorithm. An experimental study with real data is presented in Section 4 including the discussion of all results. The conclusions of this analysis are drawn in Section 5, where further research is also proposed. Finally, the acknowledgements and references sections conclude the paper.

\section{TRAVEL Time From InPut-OutPut Cumulative CounT CURVES}

Cumulative count curves are well-known tools used in different disciplines like hydraulics, hydrology or geotechnics.
Since [33] introduced them in traffic engineering for the first time, their simplicity and usefulness have been extensively proved. The works of prof. G.F. Newell represented the definitive popularization of the tool in transportation engineering. For instance [34] developed applications of queueing theory, [35] analyzed the effects of the interruption of traffic streams at signals, [36] proposed a simplified kinematic wave theory of traffic flow, or [37] analyzed delays at freeway off-ramps, in all cases using cumulative count curves.

A cumulative count curve, or $\mathrm{N}$-curve, is obtained by counting all vehicles passing over a particular location, $x$. One of these vehicles is chosen as a reference and is said to have passed at time $t=0$. From this instant, the counts of the following vehicles are accumulated over time. This allows defining the non-decreasing function $N(x, t)$ that renders the accumulated number of vehicles that have passed location $x$ by time $t$.

\section{\# FIGURE 2 \#}

Input - output diagrams are the result of depicting jointly the $\mathrm{N}$-curves measured at both ends of a section between detectors. In Fig. 2, an example of $N\left(x_{u}, t\right)$ and $N\left(x_{d}, t\right)$ is shown, where $x_{u}$ and $x_{d}$ are, respectively, the locations of the upstream and downstream detectors, defining a closed section. Typically, $N\left(x_{u}, t\right)$ is referred as the arrivals curve, while $N\left(x_{d}, t\right)$ is the departures curve. Assuming vehicle conservation and FIFO behavior, (i.e. no passing), the horizontal distance between these $\mathrm{N}$-curves at the height of any vehicle, $k$, represents the travel time of the $k^{\text {th }}$ vehicle between detectors. Note that this time includes the free flow travel time and the delay, if any. Because the analysis of delays is usually desired, the introduction of a third curve results useful. The "virtual" arrivals cumulative curve, $V\left(x_{d}, t\right)$, is obtained by translating the arrivals curve, $N\left(x_{u}, t\right)$, a length $t t_{f}$ forward in time, where $t t_{f}$ is the average free flow travel time in the section. Then, the horizontal distance between $V\left(x_{d}, t\right)$ and $N\left(x_{d}, t\right)$, at the height of any vehicle $k$, (i.e. $\left.\omega_{(k)}\right)$, yields the delay experienced by this vehicle in the section. Recall that the travel time for vehicle $k$ is composed of $t t_{f}+\omega_{(k)}$. Also note that the vertical distance between these same curves at any time $t$, (i.e. $m(t)$ ), is the vehicles' excess accumulation in the section at this instant. Obviously, $V\left(x_{d}, t\right)=N\left(x_{d}, t\right)$ if no delay exists. See [38], [39] for further details in the use of input-output diagrams.

In practice, input-output diagrams can only be used to obtain average travel times and delays across vehicles. This is not an important drawback, since average values better describe traffic conditions. There are several reasons for this limitation. First, due to passing, cumulative count curves do not truly represent individual vehicles, but positions or "labels" within the traffic stream [39] (see Section II.A). Second, loop detectors usually do not provide individual measurements, but aggregated counts and average speeds over predetermined time intervals, $\Delta t$ (as in Fig. 2). This is due to data treatment standards aimed at reducing communication and data storage needs. $\Delta t$ highly varies among countries and administrations, commonly lasting 
between 1-3 $\mathrm{min}$ in Europe and typically $30 \mathrm{~s}$ in the USA [40]. In these conditions, the procedure to obtain the average delay for a particular $\Delta t$ consists in i) computing the area $S$ enclosed between curves $V\left(x_{d}, t\right)$ and $N\left(x_{d}, t\right)$ (i.e. the total aggregated delay) and ii) dividing this area by the total number of involved vehicles. Note that $t$ will refer from now on to the instant when a particular $\Delta t$ time interval ends (i.e. discrete time units). Because $S$ can be computed in several ways, affecting different groups of vehicles, distinct average delay definitions can be obtained [41]. Although these differences are meaningful for any subsequent application, typically they are not explicitly considered [20], [21], [22]. In the present paper, arrival-based delays, $w(t)$, are of particular interest, as this type of measurements are equivalent to those directly obtained from AVI detectors. $w(t)$ is obtained from $\mathrm{N}$-curves by defining the area $S$ so that it comprises the vehicles that have exited the control section during the last $\Delta t$ (see Fig. 3a and Eq. 1). In contrast, the predicted travel time (PTT) corresponds to the expected travel time for the last vehicle that has passed over $x_{u}$ in $\Delta t$ (i.e. the last vehicle that has entered the section). The corresponding predicted delay, $p w(t)$, can be estimated from the vehicles' excess accumulation, $m(t)$, considering that a vehicle entering just after $t$ will have $m(t)$ vehicles in front of it until it can be served. Thus, the predicted delay, $p w(t)$, is computed according to Eq. 2 in Fig. $3 b$, where $\overline{p q}_{\text {out }}(t)$ is the predicted average outflow at $t$ (see Eq. 3).

\section{\# FIGURE 3 \#}

$\overline{p q}_{\text {out }}(t)$ is estimated assuming that traffic conditions will not change in the immediate future. Also, because traffic is a random process, the measurement of $\overline{p q}_{\text {out }}(t)$ is subject to statistical fluctuations. This means that the robustness of the estimation increases with the sample size or, equivalently, with the moving time window considered when computing the average. This is introduced into the calculation of $\overline{p q}_{\text {out }}(t)$ by the calibration parameter, $\delta$ (see Eq. 3), which defines the number of $\Delta t$ 's considered in the moving average. With these definitions, the variance of the sample mean estimator of $\overline{p q}_{\text {out }}(t)$ is given by $\overline{p q}_{\text {out }}(t) \cdot \sqrt{\gamma_{q} / N_{(\delta)}}$, where $\gamma_{q}$ is the index of dispersion of the flow estimation (i.e. the ratio of the variance with respect to the mean). This can be computed from a presample or simply assumed as $0.2 \div 0.3$, which are typical values in freeway traffic. In turn, $N_{(\delta)}$ is the cumulative vehicle count in the considered averaging period, of duration $\delta \Delta t$. By increasing $\delta, N_{(\delta)}$ would be larger, and the statistical significance of $\overline{p q}_{\text {out }}(t)$ would increase (i.e. smaller variance). However, by extending the averaging period, the algorithm would be slower to react to changes in the outflow, and would lose some immediacy, a relevant property of the method. Therefore, a trade-off exists in the calibration of $\delta$, yielding a more robust estimation of $\overline{p q}_{\text {out }}(t)$ that smooths out the very last changes in traffic conditions, or a more volatile one that is sensible to them. Besides, it should be noticed that, considering the central limit theorem, the estimation of $\overline{p q}_{\text {out }}(t)$ follows a normal distribution. Then, if $\overline{p q}_{\text {out }}(t)$ is substituted by its distribution in Eq. 2, the distribution of expected delays would be obtained. This would allow informing drivers, not only of the expected predicted delay, $p w$, but also of its confidence interval.

Finally, note that the method is highly independent of the freeway geometry and traffic characteristics, which may influence the bottlenecks' location but not the measurement of delays. In this regard, few empirical parameters are necessary and almost no calibration is required. Despite these advantages, some inconveniences usually appear in the practical application of the method. Next sections face these problems and propose the corresponding solutions.

\section{A. The Effects of Passing}

Theoretically, the use of input - output diagrams in queuing systems assumes FIFO behavior. In practice, passing exists in freeway traffic streams, and non-FIFO situations take place. In these cases, vehicles change their relative positions. However, if it is considered that cumulative curves do not count particular vehicles but positions (i.e. order labels that vehicles exchange with each passing maneuver), important features of the inputoutput methods such as the monotonically increasing nature of the cumulative curves are maintained [39]. This procedure prevents the use of input - output diagrams to obtain individual travel times, but average travel times will not be significantly affected, despite the passing. Note that it is possible that some of the "order labels" considered in the calculation of the average travel time for a particular $\Delta t$, actually correspond to vehicles that have exited or entered the section in the time period immediately before or after $\Delta t$. In spite of this, because the relative amount of these vehicles is small, the resulting average travel time continues being a good estimation. In this sense, [42] empirically demonstrated that freeways can be considered mostly FIFO systems and, thus, the consequences of passing in cumulative count curves, negligible.

\section{B. The Effects of Inner Section On/Off Ramps}

Difficulties arise when the surveillance configuration at a junction is that of Part b1 of Fig. 1. In such cases, two groups of vehicles can be distinguished: those that travel the whole section (i.e. all the distance between mainline detectors), and those entering or exiting through the junction, and thus, only partially covering the section. To take this into account, it would be necessary to divide the section into subsections, before and after the junction, and to construct input - output diagrams for each subsection. In the absence of mainline detectors just before and after the junction location, one solution could be to model cumulative curves given the measurements upstream (i.e. $N\left(x_{u}, t\right)$ ), downstream (i.e. $\left.N\left(x_{d}, t\right)\right)$, and the ramp counts. Newell [36] proposed a method to shift $\mathrm{N}$-curves to any desired location in between measurements. However, the method requires the flow - density relationship in the section (i.e. the fundamental diagram of traffic) as well as the a priori knowledge of the existence of bottlenecks, their precise location and their capacity. These requirements make the generalized application of Newell's 
methodology unfeasible in real time.

A simpler approximate procedure consists in directly adding the net input ramp counts (i.e. entrances minus exits) to one of the mainline detector measurements, assuming the junction to be located either at $x_{u}$ or $x_{d}$ (i.e. one of the mainline detector locations which define the target section). This means that, given a junction located at $x_{i} \in\left(x_{u}, x_{d}\right)$ with cumulative net input counts defined by $J\left(x_{i}, t\right)$, the input - output cumulative count curves for the section could be defined by one of the following options:

$$
\begin{aligned}
& \text { Input }=N\left(x_{u}, t\right)+J\left(x_{i}, t\right) \quad \text { if } x_{i} \approx x_{u} \\
& \text { Output }=N\left(x_{d}, t\right)
\end{aligned}
$$

or,

$$
\begin{aligned}
& \text { Input }=N\left(x_{u}, t\right) \\
& \text { Output }=N\left(x_{d}, t\right)-J\left(x_{i}, t\right) \quad \text { if } x_{i} \approx x_{d}
\end{aligned}
$$

On the one hand, Eq. 4 assumes $x_{i} \approx x_{u}$. On the other hand, Eq. 5 implies $x_{i} \approx x_{d}$. Any of these assumptions will introduce some bias in the average travel time estimation. This bias will be more important if the vehicles using the junction are a significant fraction of the total flow, and if their travel times are very different from the ones of vehicles covering the whole section (e.g. in case of partial congestion). In spite of this, an adequate selection between the two alternatives helps in reducing the bias of the estimation. In general, the best option is to assume the junction to be located at the closest detector location. However, if the junction is somehow in the middle and the approximate location of recurrent congestion is known, the previous criterion could be modified. Note that the alternative represented by Eq. 4 will present a higher bias if congestion is concentrated between $x_{u}$ and $x_{i}$ (because these travel times are neglected for the vehicles using the junction). In contrast, the bias will be lower if congestion concentrates between $x_{i}$ and $x_{d}$. Eq. 5 presents the opposite behavior. Therefore, the selection should be done accordingly.

\section{Detector Count Drift}

Errors in loop detector counts have been extensively reported [20], [21], [22]. Typically, detectors miss some few vehicles every $\Delta t$, without a systematic pattern and with different tendencies to undercount. This small measurement drift does not imply important drawbacks when using each detector, isolated, to measure average flows or speeds. However, inputoutput methods are used to compute the vehicles' accumulation between a pair of detectors. Even a small drift becomes significant when the count difference between detectors is accumulated over time. Thus, the error introduced by the detector drift in the estimation of average travel times can be huge. Note that vehicle accumulation is very small with respect to the total vehicle count. In fact, the accumulated count error could be larger than the vehicle accumulation itself. This paper presents a data fusion scheme to correct the count drift at detectors, enabling the of use input - output methods to estimate accurate average travel time predictions.

\section{Initialization of the Input-Output Diagram}

Despite the application of drift correction methods, a frequent reset of the input - output diagram is needed to account for the increasing bias in vehicle accumulation. At each reset, the value of the initial accumulation in the section must be estimated (see Fig. 2). However, this is not easily achieved from the measurements of loop detectors. In case of using the virtual arrivals curve $V\left(x_{d}, t\right)$ instead of $N\left(x_{u}, t\right)$ and, thus, computing delays instead of travel times, the initialization is simplified because the excess accumulation is null in free flowing traffic. Given this, it is advisable to only use input - output methods to compute delays in congested conditions. The method should turn-on just before the congestion onset, still in free flow and with null initial excess accumulation (see Section III.C). The applicability of the method only to congested conditions is not restrictive in any sense. In free flowing traffic any other methodology (e.g. spot-speed methods or direct measurements) would perform well [41]. Furthermore, real-time information is less meaningful in free flowing periods, as the uncertainty faced by the users is much less [43].

Also, note that measurement instants on curve $V\left(x_{d}, t\right)$ are displaced $t t_{f}$ time units with respect to those on curve $N\left(x_{d}, t\right)$ (see Fig. 2). Nevertheless, the construction can be simplified by considering that traffic flows evolve smoothly between consecutive $\Delta t$, and that $t t_{f}$ is generally small when compared to the precision required for the travel time estimation (e.g. $t t_{f}$ for a $2 \mathrm{~km}$ section could be $\approx 1 \mathrm{~min}$.). Then, $V\left(x_{d}, t\right)$ can be constructed simultaneously with $N\left(x_{d}, t\right)$ every $\Delta t$, neglecting the time lag between the measurements on both curves.

\section{E. $N$-Curves Linear Interpolation}

Detector counts, $n(x, t)$, are obtained at discrete time intervals, every $\Delta t$, and $N$-curves are constructed by means of linear interpolation between data points. This interpolation implies that small errors might be introduced to the average travel time estimation. However, their implications are negligible, as the aggregation periods, $\Delta t$, are usually short and traffic evolution within them can be considered gradual and smooth [41].

\section{A Data Fusion Algorithm For The Short-Term Prediction of FreEWAy TRAVEL Times}

This section describes the proposed data fusion method to correct the drift in input - output count curves by using AVI direct travel time measurements. This includes the temporal and spatial alignment of the different sources of information, the description of the turn-on and turn-off conditions to ensure the periodic reset of the cumulative curves, the data fusion algorithm itself, and the computation of the predicted travel time from the input - output diagrams. The flowchart in Fig. 4 summarizes the main steps of the methodology. A detailed explanation of all of them is provided in the next subsections.

\section{\# FIGURE 4 \#}




\section{A. Data Inputs to the Algorithm}

The proposed method requires two types of real-time inputs. First, data coming from loop detectors, which generally include the time-mean speed, $v$, vehicle count, $n$, and detector occupancy, $\rho$, over an aggregation period, $\Delta t$. Second, direct travel time measurements obtained from AVI technologies, which consist in the arithmetic average of the measured travel times on a target section during an aggregation period, $\Delta T$.

The duration of aggregation periods responds to a trade-off between the robustness in the estimation of average values (which grows with the sample size), and their updating frequency (which allows tracking changes in traffic conditions rapidly). In general, $\Delta T>\Delta t$, because only a fraction of vehicles can be automatically identified by AVI technologies, while loop detectors yield comprehensive measurements. Short AVI 's $\Delta T$ imply large fluctuations in the average direct travel time estimation and high bias due to the stronger effect of outliers, especially in off-peak demand periods when traffic demand is low (i.e. small sample size). This is why $\Delta T$ is typically long, ranging between $5 \div 15 \mathrm{~min}$. In contrast, due to the larger sample size at loop detectors, shorter $\Delta t$ 's suffice to obtain robust average speed estimations. In addition, vehicle count, $n$, and detector occupancy, $\rho$, are cumulative measurements, where the sample size does not affect the robustness of the measurement. Given this context, $\Delta t$ frequently ranges between $0.5 \div 3 \mathrm{~min}$. This difference between the duration of aggregation periods (i.e. $\Delta T>\Delta t$ ) implies that loop detectors are able to provide a quicker response to changes in traffic conditions if compared to AVI measurements. This property is exploited in the proposed method, where it is assumed that $\Delta T$ is an integer multiple of $\Delta t$. This condition, which is typically fulfilled, facilitates the temporal alignment of both data types.

Regarding the spatial distribution of data, Fig. 5 sketches the typical freeway surveillance layout. AVI devices, like Bluetooth detectors or LPR devices, are usually installed on overhead gantries and are fewer in comparison with loops. In this context, direct travel time measurements define the target freeway stretch for which real-time information will be provided (i.e. between AVI devices). Then, loop detectors divide this stretch into $k$ sections, which may range from 500 $\mathrm{m}$ to $2 \mathrm{~km}$. It is assumed that the location of AVI devices coincides with that of one loop detector, which is frequently the case.

\section{\# FIGURE 5 \#}

\section{B. The Data Fusion Method to Correct Detector Drift}

Typically, the detector drift between a pair of loop detectors is corrected by imposing that, over the long term, the input count must be equal to the output count. Note that the change in vehicle accumulation is negligible when compared to the long-term counts. So, the long-term drift correction factor, $\beta_{i}$, where the subscript $i$ defines a section delimited by its upstream, $i_{u}$, and downstream, $i_{d}$, loop detectors, is computed as:

$$
\beta_{i}=\frac{\sum_{t=0}^{\text {long term }} \hat{n}\left(i_{d}, t\right)}{\sum_{t=0}^{\text {long term }} \hat{n}\left(i_{u}, t\right)}
$$

Where $\hat{n}$ refers to the raw counts at the upstream and downstream detectors, and taking into account that a minimum of $24 \mathrm{~h}$ is usually considered by "long term". Then, the upstream count, corrected for the long-term drift, $n\left(i_{u}, t\right)$, would be:

$$
n\left(i_{u}, t\right)=\beta_{i} \cdot \hat{n}\left(i_{u}, t\right)
$$

Note that the long-term correction factor is only applied to arrivals (i.e. to the counts at the upstream detector), while departures (i.e. counts at the downstream detector) are assumed to be correct. The opposite approach would lead to identical results. This assumption is maintained throughout the paper, so that departure cumulative curves are considered correct, and only (virtual) arrival curves are rectified to account for the drift.

This long-term drift correction is not enough to achieve an accurate travel time estimation. The drift factor typically varies in the short term, especially in congested conditions, so that the long-term average does not suffice to correct the curves. Henceforth, long-term drift correction is considered as default and an additional short-term correction needs to be applied on top of that.

The proposed method uses direct travel time measurements to correct the short-term drift in loop detector counts. The concept of the data fusion scheme is simple: the accurate (but delayed) direct travel time measurement is compared to an equivalent ATT estimation obtained from the input-output diagram. The direct measurement is assumed to be the ground truth, and the virtual arrivals curve, $V\left(i_{d}, t\right)$, is modified until both travel time estimations coincide. The corrected virtual arrivals curve, $V^{*}\left(i_{d}, t\right)$, is then used to compute the PTT, as in Eq. 2 in Fig. $3 b$.

The main difficulty of the method consists in obtaining equivalent estimations so that they are comparable. To this end, both travel time measurements need to be aligned temporally and spatially. Consider $\widehat{t} t(t)$ to be the direct ATT measurement obtained at $t=T$ (i.e. at the end of a $\Delta T$ time period) for a particular freeway stretch composed of $k$ sections (i.e. $i=$ $1,2, \ldots, k)$. Note that the " $`$ " notation is used to describe raw measured variables throughout the paper. $\widehat{t t}(t)$ can be expressed as the free flow travel time, $t t_{f}$, plus the total delay in the stretch, $\widehat{w}(t)$ (see Eq. 8):

$$
\widehat{t t}(t)=t t_{f}+\widehat{w}(t)
$$

The spatial alignment is achieved by dividing the total delay from direct measurement, $\widehat{w}(t)$, between the sections that compose the stretch. To that end, $\widehat{w}(t)$ needs to be divided into $k$ values of $\widehat{w}_{i}\left(t^{\prime}\right)$, yielding the direct travel time estimation for section $i$. This is done proportionally to the relative magnitude of the raw partial delays from input-output curves, $w_{i}\left(t^{\prime}\right)$ (see Eq. 9). 


$$
\widehat{w}_{i}\left(t^{\prime}\right)=\frac{w_{i}\left(t^{\prime}\right)}{\sum_{j=1}^{k} w_{j}\left(t^{\prime}\right)} \cdot \widehat{w}(t) \quad(\text { when } t=T)
$$

Note that, because direct measurements are ATT, $w_{i}\left(t^{\prime}\right)$ need to be equivalent arrival-based average delays computed from input-output curves (as in Eq. 1. in Fig. 3a). In addition, time moving coordinates (denoted with an accent, e.g. $t^{\prime}$ ) are needed, because ATT is a trajectory-based measurement and, thus, a temporal alignment is necessary. Fig. 6 and Eq. 10 describe the computation process of $w_{i}\left(t^{\prime}\right)$ from input-output curves. Note that this computation process must start from the most downstream section (i.e. $i=1$ ), for which the time alignment is met by default (i.e. $t=t^{\prime}$ ) and the time lag is null.

\section{\# FIGURE 6 \#}

Then, every $\Delta T$ (i.e. when $t=T$; this will be the updating period for the short-term drift correction factor) two equivalent estimations for the sectional delay are available: $w_{i}\left(t^{\prime}\right)$ from input-output curves, which includes the drift error, and $\widehat{w}_{i}\left(t^{\prime}\right)$, a more accurate estimation from direct measurements.

The corrected virtual arrivals curve, $V^{*}\left(i_{d}, t\right)$, is obtained by modifying the counts of the virtual arrivals curve, $V\left(i_{d}, t\right)$, as in Eq. 11:

$$
V^{*}\left(i_{d}, t\right)=\alpha_{i}(t) \cdot V\left(i_{d}, t\right)
$$

Where $\alpha_{i}(t)$ is the short-term drift correction factor, obtained by imposing that $w_{i}^{*}\left(t^{\prime}\right)=\widehat{w}_{i}\left(t^{\prime}\right)$, being $w_{i}^{*}\left(t^{\prime}\right)$ the corrected delay corresponding to $V^{*}\left(i_{d}, t\right)$. The solution can be achieved through numerical optimization, setting a small tolerance, $\tau$, for the final difference to ensure a fast convergence.

Note from Figure 6 that the correction in Eq. 11 is applied to the different counts existing between $t^{\prime}-\Delta T$ and $t^{\text {current }}$. This implies the existence of two levels of corrections. If the count $\in\left(t^{\prime}-\Delta T, t^{\prime}\right)$, the correction is said to be final, because this will not be modified in future time steps. Otherwise, if the count $\in\left(t^{\prime}, t^{\text {current }}\right)$, the correction is temporal and could be further modified in next iterations.

\section{The Algorithm Turn-on and Turn-off Conditions}

While free flowing conditions prevail, delays and excess accumulation are null, the data fusion algorithm is off, and a default free flow travel time is enough to feed the real-time information system. The algorithm should turn-on just before the appearance of delays. With this objective, turn-on conditions must focus on the quick detection of the congestion onset. Being delayed measurements, direct travel time measurements are not useful to this end.

Turn-on conditions are defined based on loop detector measurements at $i_{u}$ and $i_{d}$, the upstream and downstream detectors of section $i$ (see Eqs. 12 to 14). The fulfillment of any of these conditions suffices to activate the algorithm at the instant $t-\Delta t$, from where cumulative curves are initialized with null excess accumulation as described in Section II.D.

$$
\left\lceil v\left(i_{u}, t\right)\right\rceil \leq v_{r e f}
$$

$$
\begin{array}{r}
\left\lceil v\left(i_{d}, t\right)\right\rceil \leq v_{r e f} \\
\frac{\partial\left\lfloor V\left(i_{d}, t\right)\right\rfloor}{\partial t}>\frac{\partial\left\lceil N\left(i_{d}, t\right)\right\rceil}{\partial t}
\end{array}
$$

Eqs. 12 and 13 detect congestion at the detector locations by measuring a speed, $v$, lower than a given threshold, $v_{r e f}$. $v_{r e f}$ should be calibrated in any particular application of the algorithm. Generally, a low percentile of the free flow speed distribution suffices (e.g. $1^{\text {st }}$ quartile of the distribution of measured speeds during free flowing periods, typically around $100 \mathrm{~km} / \mathrm{h}$ in multilane freeways). The \ 1 brackets in Eqs. 12 and 13 indicate that an upper bound of the speed estimation is considered, in order to account for the statistical fluctuations in the speed measurement. This upper bound can be computed as the upper limit of a confidence interval of the speed estimation. This is:

$$
\lceil v(i, t)]=v(i, t)+\text { prob.level } \cdot \frac{c v_{v} \cdot v(i, t)}{\sqrt{n(i, t)}}
$$

Where $c v_{v}$ is the coefficient of variation of the speed measurements (i.e. the standard deviation over the mean, which can be obtained from a pre-sample) and $n$ is the vehicle count in the time period considered (i.e. the sample size). The prob.level defines the significance of the confidence interval (e.g. $68 \%$ for a prob.level $=1$ ).

In contrast, Eq. 14 detects congestion within the section by measuring that inflows, $\frac{\partial\left[V\left(i_{d}, t\right)\right]}{\partial t}$, are higher than outflows, $\frac{\partial\left[N\left(i_{d}, t\right)\right]}{\partial t}$ (i.e. growing excess accumulation). Note that Eq. 14 is equivalent to $\frac{\partial m_{i}(t)}{\partial t}>0$. In order to account for the statistical fluctuations in the flow estimation and to avoid false positives, a lower bound is considered for the inflows, and an upper bound for the outflows. Again, these can be computed from the confidence interval in the flow estimation, as in Eqs. 16 and 17:

$$
\begin{aligned}
& \frac{\left.\partial \mid V\left(i_{d}, t\right)\right\rfloor}{\partial t}=\frac{\partial V\left(i_{d}, t\right)}{\partial t}-\text { prob. level } \cdot \frac{\partial V\left(i_{d}, t\right)}{\partial t} \cdot \sqrt{\frac{\gamma_{q}}{n\left(i_{u}, t\right)}} \\
& \frac{\partial\left[N\left(i_{d}, t\right)\right]}{\partial t}=\frac{\partial N\left(i_{d}, t\right)}{\partial t}+\text { prob.level } \cdot \frac{\partial N\left(i_{d}, t\right)}{\partial t} \cdot \sqrt{\frac{\gamma_{q}}{n\left(i_{d}, t\right)}}
\end{aligned}
$$

Recall that $\gamma_{q}$ is the index of dispersion of the flow estimation (i.e. the ratio of the variance with respect to the mean).

On its behalf, there is only one turn-off condition. This is based on the achievement of null excess accumulation (i.e. $\left.m_{i}(t)=0\right)$. However, due to the statistical fluctuations in the count measurement, a higher than zero threshold needs to be set. This is formulated in Eq. 18, noting that $\sqrt{\gamma_{q} \cdot n(i, t)}$ is the statistical variability of the count estimation for a $68 \%$ confidence level.

$$
\begin{aligned}
& m_{i}(t)=V^{*}\left(i_{d}, t\right)-N\left(i_{d}, t\right)< \\
&<\sqrt{\gamma_{q} \cdot\left[n\left(i_{u}, t\right)+n\left(i_{d}, t\right)\right]}
\end{aligned}
$$




\section{IMPLEMENTATION OF THE ALGORITHM WITH REAL DATA ON THE AP7 FREEWAY IN SPAIN}

The data used to test the algorithm was measured on April $25^{\text {th }}, 2010$, in a 3-lane stretch of the AP7 freeway in its southbound direction towards Barcelona, Spain. This was a sunny Sunday with some light evening congestion in the AP7 due to the large demand returning the city after the weekend on the coast. For the rest of the day traffic was free flowing. The layout of the test site is illustrated in Fig. 7. The target stretch, with a length of $12.8 \mathrm{~km}$, is limited by AVI devices (i.e. Bluetooth detectors) at both ends. In addition, there are doubleloop detectors at the same location, plus one more in between (i.e. at Kilometer Post - K.P. 113.9), which divides the stretch into two sections $(i=1,2)$. Section $i=2$ (i.e. the upstream one) contains the St. Celoni junction at K.P. 111.6, near its downstream end. Entrances and exits through this junction are monitored. This layout configuration is thus adequate to test the goodness of the proposed method to obtain short-term travel time predictions.

\section{\# FIGURE 7 \#}

Vehicle counts, $n$, and time-mean speeds, $v$, are available for time aggregations of $\Delta t=3 \mathrm{~min}$ at loop detector locations. In addition, the net input counts at the junction are also computed for the same $\Delta t$. These are transferred to the nearest detector (i.e. at K.P. 113.9). Because this is the downstream detector of Section $i=2$, the junction's net input counts must be subtracted from the detector counts (see Eq. 5).

Average AVI direct travel time measurements are available every $\Delta T=6 \mathrm{~min}$. In spite of this, and for the only purpose of validating the method, individual vehicles' travel time measurements were recorded. These data allow obtaining the ground truth predicted travel time, $\widehat{p t t}(t)$, defined as the departure-based travel time of those vehicles entering the target stretch between $t$ and $t+\Delta t$ (i.e. the travel time actually experienced by the vehicles that receive the real-time information at $t$ ).

All relevant parameters and variables of the method are summarized in Table 1. Note that only the free flow travel time, $t t_{f}$, and the long-term drift correction factor, $\beta$, are highly dependent on the highway section under analysis. The rest of parameters exhibit lower sensitivities to new road contexts, and some of them are even insensitive to the context, so that the proposed values may be set as defaults. For these parameters, calibration might be omitted if necessary.

\section{\# TABLE 1 \#}

\section{A. Obtained Results and Discussion}

Fig. 8 shows the performance of the proposed method for the evening light congestion episode, between 6 and $10 \mathrm{pm}$. The predicted travel times from the method are compared against the ground truth travel times of those vehicles that would have received the real-time information. The results in case of simply disseminating the direct AVI measurements (i.e. the current practice) or those obtained using the midpoint spot-speed algorithm (i.e. travel times from speed measurements at loop detectors assuming uniform speeds until the midpoint location between detectors [44]) are also shown. Table 2 quantitively summarizes the mean and maximum errors.

\section{\# FIGURE 8 \# \\ \# TABLE 2 \#}

The results shown in Fig. 8 and Table 2 demonstrate that the proposed algorithm satisfactorily accomplishes its objectives, and provides drivers with a better prediction of their travel times over the freeway stretch, improving the information given by the dissemination of direct measured travel times or by the common midpoint spot-speed algorithm. Fig. 8 clearly shows how the midpoint algorithm overreacts to travel time changes. Regarding AVI direct measurements, they fail in predicting the evolution of travel times due to the delay in reporting the information. This implies large errors when travel times change rapidly, especially at congestion dissolve episodes. In contrast, short-term predicted travel times are able to respond quicker, providing better travel time predictions to drivers, with lower maximum errors in rapid evolving conditions.

However, it can be seen from Fig. 8 that the short-term predictions obtained from the proposed method still show some delay. This is due to the adoption of a long moving average interval in the computation of $\overline{p q}_{\text {out }}$ (i.e. $\delta=7$; see Table 1). As discussed in Section II, longer averaging periods imply more delay in the information, in exchange for a more robust estimation. Shorter averaging periods (i.e. lower $\delta \mathrm{s}$ ) would reduce delay in the short-term predictions, but would increase their fluctuations. For this particular application of the method, $\delta=7$ turned out to be optimal in terms of reducing the average and maximum errors.

In fact, it has been found that in this case study the fluctuations in the predicted travel times were larger than expected. Some large fluctuations remain even considering a long averaging interval in the computation of $\overline{p q}_{\text {out }}$ (e.g. the underestimation around 19:15, or the overestimation around 19:45). Further analysis of these periods unveiled that these fluctuations were related to abnormal net counts at the junction, representing a significant fraction of the main trunk detector counts. While the junction counts were probably erroneous during these periods, this could not be definitively proved. In any case, these results highlight that the proposed method is sensitive to large changes in the vehicle counts affecting $\overline{p q}_{\text {out }}$. Therefore, the method handles well small random drift errors in the loop detectors but it will produce bad estimations in case of undetected detector failure (e.g. partial data losses).

Another issue that implies bias in the present case study is the assumption of the net junction counts taking place at the downstream detector of Section $i=2$. Note that this affects the computation of $\overline{p q}_{\text {out }}$, and the predicted travel time is sensitive to this average outflow (see Fig. 3 and Eq. 2). Even if there is a small distance between the junction and the detector, in congested conditions the time lag between both can be larger 
than $\Delta t$, implying a misalignment in time. In addition, the loop detector outflow in congested conditions can be small, so that the junction counts could be a significant fraction of it. All this together, might cause fluctuations in the predicted travel time. In order to avoid this type of errors, mainline detectors before and after the junction, instead of at on/off ramps would be preferred.

Finally, it is worth noting that in case of more severe congestion, errors and information delay of directly disseminated AVI measurements would grow proportionally to the delay, while the errors of the proposed method would be similar to the ones in the present case study, and thus lower in relative terms. Also, the benefits of using the method would be more significant if AVI detectors were further apart, which is the case in most freeways in Spain and around the world.

\section{CONCLUSIONS AND FURTHER RESEARCH}

Freeway travel time will continue being amongst drivers' most valuable traffic information. With all the technological evolution and the development of on-board positioning and communication devices, direct travel time measurements are becoming a popular data source for traffic information systems. Nevertheless, the real-time dissemination of these measured travel times has some limitations, like the delay in the information (i.e. measured travel times are past information) or the lack of data for some periods with very small sample sizes. In this context, this paper proposes a methodology to obtain more accurate predicted travel times by fusing data from traditional loop detectors and direct travel time measurements. Both data sources are commonly available, even in low surveillance environments, which supports the immediate implementation of the method without significant extra costs in most cases. The fact that a relatively low sample of direct measurements suffices to apply the method, confirms this assertion.

The proposed travel time estimation methodology is based on the use of input-output cumulative count curves to determine the vehicles' accumulation in a freeway section. This means that the method requires "closed" freeway sections, in the sense that all inflows and outflows need to be monitored (i.e. upstream and downstream detectors in the main freeway trunk, as well as inner junctions). From the vehicles' accumulation, predicted travel times are computed using the principle of the vehicles' conservation. The derivation of travel times from cumulative count curves is not new. However, input-output methods have not been used in practice so far, mainly due to the flawed results caused by the loop detector drift. In this sense, the main contribution of the present paper is the design of a data fusion scheme aimed at correcting the detector drift in cumulative count curves. Direct travel time measurements, supplied either by AVI systems or tracking devices, can be used to this end.

The benefits of the proposed methodology with respect to the simple dissemination of directly measured travel times include a quicker detection of travel time changes, a higher updating frequency and the possibility of obtaining partial travel times for sections within the target stretch. These properties greatly contribute to reduce the errors of the disseminated information, especially the maximum errors that arise in rapidly evolving traffic conditions (i.e. congestion onset / dissolve episodes). These benefits would be larger in situations of severe congestion with large delays and in long freeway stretches between AVI measurements. In free flowing conditions, the proposed method could be turned off, as all types of travel time estimations suffice (e.g. direct measurements, or travel times estimated from the speed measurement at loop detectors).

The methodology has been validated with real data from the AP7 freeway towards Barcelona (Spain). Travel times from Bluetooth detectors have been used as AVI direct measurements. Results show that the predicted travel times obtained with the proposed methodology are able to better match the real travel times that the drivers receiving the information will experience, in comparison with the current practice of directly disseminating AVI measurements or using the common midpoint spot-speed method from loop measurements. The mean average percentage error was reduced from $17.0 \%$ and $29 \%$, respectively, to $10.8 \%$. These benefits could be observed even in a scenario with light congestion, despite the methodology being especially suited for medium to severe congestion episodes. With the available real data, the mean and maximum absolute errors achieved with the predicted travel times (i.e. $1.24 \mathrm{~min}$ and $4.5 \mathrm{~min}$ respectively) represented approximately $11 \%$ and $33 \%$ of the experienced travel times. The maximum errors in the case study resulted from fluctuations in the predicted travel time, which could be attributed to detector malfunctioning or data loss. This issue highlights that the method deals well with the random detector drift (which invariably affects all detectors), but is sensitive to more severe detector failures. In this respect, a complementary algorithm to detect detector malfunctioning, so that the lost data could be replaced, is left as further research. In such situation, the method would be even more accurate, not only by reducing the maximum errors, but also because the calibration parameters could then prioritize immediacy in reporting travel time changes with respect to smoothing artificial fluctuations.

\section{ACKNOWLEDGMENT}

The authors acknowledge the work of Mr. Enrique Jiménez in the AP7 data treatment. This research would not have been possible without the collaboration of Abertis, and especially of Mr. Joan Altarriba in the data acquisition process.

\section{REFERENCES}

[1]. Hall, R. W. (1993). Non-recurrent congestion: How big is the problem? Are traveler information systems the solution? Transportation Research Part C: Emerging Technologies 1(1), 89-103.

[2]. Barceló, J., L. Montero and L. Marquès. (2010) Travel Time Forecasting and Dynamic OD Estimation in Freeways Based on Bluetooth Traffic Monitoring. Proc. of the 89th Annual Meeting of the Transportation Research Board. Washington D.C.

[3]. Longfoot, J. (1991). An Automatic Network Travel Time SystemANTSS. Proceedings of the 1991 Vehicle Navigation and Information Systems Conference, 2, 20-23 October 1991, Dearborn, Michigan, 10531061. 
[4]. Nishiuchi, H., K. Nakamura, S. Bajwa, E. Chung and M. Kuwahara. (2006). Evaluation of travel time and OD variation on the Tokyo Metropolitan Expressway using ETC data. Research into Practice: 22nd ARRB Conference Proceedings Information, Australian Road Research Board.

[5]. Soriguera, F., D. Rosas and F. Robusté. (2010). Travel Time Measurement in Closed Toll Highways. Transportation Research Part $B$ 44(10), 1242-1267.

[6]. Buisson, C. (2006). Simple traffic model for a simple problem: sizing travel time measurement devices. Transportation Research Record: Journal of the Transportation Research Board 1965, 210-218.

[7]. van Hinsbergen, C. P. I. J., J. W. C. van Lint and F. M. Sanders (2007). Short Term Traffic Prediction Models. Proceedings of the 14th World Congress on Intelligent Transport Systems (ITS), 2007, Beijing, 1-18.

[8]. Coifman, B., and M. Cassidy. (2002) Vehicle reidentification and travel time measurement on congested freeways. Transportation Research Part A 36(10), 899-917.

[9]. Coifman, B. and E. Ergueta. (2003). Improved vehicle reidentification and travel time measurement on congested freeways. ASCE Journal of Transportation Engineering 129(5), 475-483.

[10]. Coifman, B. and S. Krishnamurthya. (2007). Vehicle reidentification and travel time measurement across freeway junctions using the existing detector infrastructure. Transportation Research Part C 15(3), 135-153.

[11]. Kuhne, R. and S. Immes. (1993) Freeway control systems using sectionrelated traffic variable detection. Proc. of Pacific Rim TransTech Conference 1, 56-62. ASCE.

[12]. Abdulhai, B. and S.M. Tabib. (2003). Spatio-Temporal InductancePattern Recognition for Vehicle Reidentification. Transportation Research Part C 11(3-4), 223-239.

[13]. Kwon, T. M. (2006) Blind deconvolution processing of loop inductance signals for vehicle reidentification. Proc. of the 85th Annual Meeting of the Transportation Research Board. Washington D.C.

[14]. Herrera, J. C., D. B. Work, R. Herring, X. Ban, Q. Jacobson and A. M. Bayen. (2010). Evaluation of traffic data obtained via GPS-enabled mobile phones: The Mobile Century field experiment. Transportation Research Part C 18(4), 568-583.

[15]. Yim, Y. (2003). The State of Cellular Probes. Technical Report. July. Berkeley: Institute of Transportation Studies, University of California.

[16]. Kaufmann, S., B. S. Kerner, H. Rehborn, M. Koller and S. L. Klenov (2018). Aerial observations of moving synchronized flow patterns in over-saturated city traffic. Transportation Research Part C: Emerging Technologies 86, 393-406.

[17]. Soriguera, F. and F Robusté. (2011a). Requiem for freeway travel time estimation methods based on blind speed interpolations between point measurements. IEEE Transactions on Intelligent Transportation Systems. $12(1), 291-297$.

[18]. Coifman, B. (2002). Estimating travel times and vehicle trajectories on freeways using dual loop detectors. Transportation Research Part A 36(4), 351-364.

[19]. Treiber, M. and D. Helbing. (2002). Reconstructing the spatio-temporal traffic dynamics from stationary detector data. Cooper@tive Tr@nsport@tionDyn@mics 1,3.1-3.24.

[20]. Nam, D. H., and D.R. Drew. (1996) Traffic dynamics: Method for estimating freeway travel times in real time from flow measurements. ASCE Journal of Transportation Engineering 122(3), 185-191.

[21]. Oh, J.S., R. Jayakrishnan and W. Recker. (2003). Section travel time estimation from point detection data. Paper presented in the 82nd Transportation Research Board Annual Meeting. Washington D.C.

[22]. van Arem, B., M.J.M. van der Vlist, M.R. Muste and S.A. Smulders. (1997). Travel Time Estimation in the GERIDIEN Project. International Journal of Forecasting 13, 73-85.

[23]. Ambühl, L. and M. Menéndez. (2016). Data fusion algorithm for macroscopic fundamental diagram estimation. Transportation Research Part C 71, 184-197.

[24]. Deng, W., H. Lei, and X. Zhou. (2013). Traffic state estimation and uncertainty quantification based on heterogeneous data sources: A three detector approach. Transportation Research Part B 57, 132-157.
[25]. Nantes, A., D. Ngoduy, A. Bhaskar, M. Miska, and E. Chung. (2016). Real-time traffic state estimation in urban corridors from heterogeneous data. Transportation Research Part C 66, 99-118.

[26]. Sun, Z., W. Jin, and S. Ritchie. (2017). Simultaneous estimation of states and parameters in Newell's simplified kinematic wave model with Eulerian and Lagrangian traffic data. Transportation Research Part $B$ 104, 106-122.

[27]. Chen, H, and H. Rakha. (2016). Multi-step prediction of experienced travel times using agent-based modelling. Transportation Research Part C 71, 108-121.

[28]. El Faouzi, N. and M. Maurin. (2007). Reliability of travel time under lognormal distribution: methodological issues and path travel time confidence derivation. Transportation Research Board 86th Annual Meeting. Washington D.C.

[29]. Soriguera, F. and F. Robusté. (2011b). Highway travel time accurate measurement and short-term prediction using multiple data sources. Transportmetrica, 7(1), 85-109.

[30]. Van Lint, J. W. C., S. P. Hoogendoorn and H. J. van Zuylen. (2005). Accurate freeway travel time prediction with state-space neural networks under missing data. Transportation Research Part C 13(5-6), 347-369.

[31]. Gu, Y., W. Lu, L. Qin, M. Li and Z. Shao. (2019) Short-term prediction of lane-level traffic speeds: a fusion deep learning model. Transportation Research Part C 106, 1-16.

[32]. Karlaftis, M. G. and E. I. Vlahogianni. (2011). Statistical methods versus neural networks in transportation research: differences, similarities and some insights. Transportation Research Part C 19(3), 387-399.

[33]. Moskowitz, K. (1954). Waiting for a gap in a traffic stream. Proc. Highway Res. Board 33, 385-395.

[34]. Newell, G. F. (1982) Applications of queuing theory, 2nd ed. Chapman and Hall, London.

[35]. Newell, G. F. (1988). Theory of Highway Traffic Signals, Institute of Transportation Studies, UC Berkeley.

[36]. Newell, G. F. (1993). A simplified theory of kinematic waves in highway traffic, I general theory, II queuing at freeway bottlenecks, III multidestination flows. Transportation Research Part B 27(1), 281-313.

[37]. Newell, G. F. (1999). Delays caused by a queue at a freeway exit ramp. Transportation Research Part B 33(5), 337-350.

[38]. Daganzo, C. F. (1983) Derivation of delays based on input-output analysis. Transportation Research Part A 17(5), 341-342.

[39]. Daganzo, C. F. (1997). Fundamentals of Transportation and Traffic Operations. Pergamon. Elsevier Science Ltd.

[40]. Soriguera, F. and F. Robusté. (2011c). Estimation of traffic stream spacemean speed from time aggregations of loop detector data. Transportation Research Part C 19(1), 115-129.

[41]. Soriguera, F. (2016). Highway travel time estimation with data fusion. Springer Tracts on Transportation and Traffic, 11. Springer-Verlag Berlin Heidelberg.

[42]. Muñoz, J. C. and C. F. Daganzo. (2002). The bottleneck mechanism of a freeway diverge. Transportation Research Part A 36(6), 483-505.

[43]. Soriguera, F. (2014). On the Value of highway travel time information systems. Transportation Research Part A 70, 294-310.

[44]. Soriguera, F. and F. Robusté. (2013). Freeway travel-time information: design and real-time performance using spot-speed methods. IEEE Transactions on Intelligent Transportation Systems 14(2), 731 - 742. 


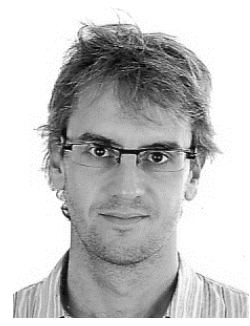

Francesc Soriguera received the M.Eng. and $\mathrm{Ph} . \mathrm{D}$. degrees in civil engineering from UPC-BarcelonTech, Barcelona, Spain, in 2004 and 2010, respectively.

In 2007 and 2012, he was a Visiting Scholar with the University of California at Berkeley, and in 2015 at TØI, the Institute of Transportation Economics - Norwegian Center for Transport Research. He is currently an Associate Professor with the Department of Civil and Environmental Engineering, UPC-BarcelonaTech, member of the BIT-Barcelona Innovative Transportation research group, and the academic coordinator of the Master's degree in Supply Chain, Transport and Mobility Management (UPC-BarcelonaTech). His current research interests include the improvement of freeway traffic with the presence of autonomous and connected vehicles and vehicle sharing schemes in urban mobility.

Dr. Soriguera received the Abertis Research Award (2010) for his research regarding freeway travel time estimation methods, the UPC-BarcelonaTech outstanding Ph.D. award (2010), the Young Researcher Award (CIT conference 2012), and he was shortlisted in the prestigious International Transport Forum Young Researcher Award (OECD, 2014).

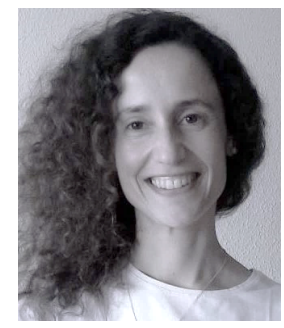

Margarita Martínez-Díaz received the M.Eng. and Ph.D. degrees in civil engineering from the Universidade da Coruña (UDC), A Coruña, Spain, in 2002 and 2018, respectively.

She is currently an Assistant Professor at the Civil Engineering School (Universidade da Coruña) and a regular Visiting Professor at the Hochschule Magdeburg-Stendal (Germany). She is member of the Agrupación Estratéxica CITEEC (Universidade da Coruña).

In 2015 and 2017, she was a Visiting Researcher at the UPCBarcelonaTech under the supervision of Prof. Soriguera, with whom she continues to collaborate. Her current research interests focus on dynamic traffic management including connected and automated vehicles.

Dr. Martínez-Díaz received the Dragados y Construcciones Award and the Tracim Award (2003) for her Master's Degree Final Project. Also, she received the second Prize of the Campus FIT of the Foro de Ingeniería del Transporte (2017) for the preliminary stages of her Ph.D dissertation. More recently, her doctoral thesis was awarded with both the International and the National Abertis research awards in management of Transportation Infrastructures. 